\title{
LOS CONCEPTOS PAMPA/CH'URU EN LA MANUFACTURA DE LAS FAJAS CONFECCIONADAS POR MUJERES AYMARA DEL NORTE CHILENO
}

\author{
THE CONCEPTS PAMPA/CH'URU IN THE MANUFACTURING OF \\ BELTS MADE BY AYMARA WOMEN OF NORTHERN CHILE
}

\author{
Vivian Gavilán Vega ${ }^{1}$, Iza Álvarez $^{1}$ y Katherine Cisternas ${ }^{1}$
}

\begin{abstract}
El artículo presenta resultados de investigación acerca de las bases conceptuales que sustentan los diseños de las fajas del altiplano del norte chileno. Específicamente, aquellas que subyacen en los tejidos elaborados en las comunidades de Isluga y Cariquima. La lectura realizada, en conjunto con las tejedoras, identificó los principales componentes simbólicos que orientan la manufactura de esta pieza del vestido. Las fajas se clasifican en dos tipos: fajas pampa y fajas ch'uru. Esta tipificación ha sido identificada en otros estudios de las tradiciones textiles del sur andino. Se retoman los datos por estos señalados y se agregan nuevos antecedentes respecto de la relación que las mujeres establecen entre diseño y uso, lo cual permite comprender significados situados en el dominio religioso en contextos de dominación colonial. Se identifican tres criterios utilizados por las tejedoras en la planificación de los diseños: la direccionalidad del hilado y torcido, el color y el número de chinu o nudos.
\end{abstract}

Palabras claves: textiles andinos, memoria colectiva, saberes pueblos originarios.

The article presents results of research on the conceptual basis that support designs of highland belts of northern Chile. Specifically, those underlying the textiles developed in the communities of Isluga and Cariquima. The interpretation performed in conjunction with the weavers, identified the main symbolic components that guide the manufacture of this piece of dress. The belts are classified into two types: belt pampa and belt ch'uru. This classification has been identified in other studies of the textile traditions of the southern Andes. This data is restudied and new information is added about the relationship that women establish between design and use, which allows the understanding of the meanings placed in the religious domain within the context of colonial domination. Three criteria used by women weavers in the planning of designs were identified: directionality and twist of the yarn, the color, and the number of chinu or knots.

Key words: Andean textiles, collective memoir, knowledgement of native people.

Descodificar los mensajes de los tejidos elaborados por mujeres y hombres descendientes de los pueblos originarios residentes en el norte chileno y descifrar las ideas que subyacen en cada pieza, no es tarea fácil. A pesar de los avances logrados hacen falta más estudios que nos permitan mejores y mayores aproximaciones. Se vuelve una tarea urgente debido a la escasez de personas que hoy practican esta actividad y dado el pequeño grupo de expertas-os y conocedoras-es de las bases técnicas, lingüísticas y epistémicas del hacer textil.

La pérdida de los saberes textiles tiene un efecto inconmensurable no solo para las familias de origen indígena sino para la humanidad en su conjunto. Tanto desde el punto de vista tecnológico como desde la perspectiva conceptual los tejidos forman parte del patrimonio cultural, material e inmaterial de los pueblos originarios y son el soporte material que alberga la memoria colectiva de los mismos. Configuran, así, una manifestación particular de conocer el mundo y un instrumento de comunicación. En este contexto, este trabajo intenta avanzar en el conocimiento de las bases conceptuales que sustentan la manufactura de las fajas en las comunidades de Isluga y Cariquima, ubicadas en la zona altiplánica de la actual región de Tarapacá, en el norte chileno.

Nos interesa abordar el estudio de esta pieza de la vestimenta de mujeres y hombres andinos, pues su uso -ya sea en la cotidianidad o en las festividades-y los significados asociados nos permiten comprender un lenguaje particular. Su confección obedece a un esquema conceptual aplicado por las tejedoras y transmitido oralmente de generación en generación. Basándonos en la premisa de que la práctica textil, en tanto soporte de la memoria colectiva de los

1 Departamento de Antropología, Universidad de Tarapacá, 18 de Septiembre 2222, Casilla 6-D, Arica-Chile. viviangav@yahoo.com 
pueblos originarios, entrega información valiosa de un sistema histórico-cultural de comunicación, buscamos aproximarnos a las formas de conocer y estar en el mundo de las tejedoras.

La revisión de varias colecciones de fajas, las entrevistas y conversaciones con mujeres y hombres, y los antecedentes bibliográficos nos condujeron a un conjunto de datos significativos. Los cuadernos de campo y las fichas de registro elaboradas con las tejedoras, entre 1991 y 1993, fueron de importancia central. Los relatos acopiados con posterioridad triangularon la información. Los resultados obtenidos entregan nuevos antecedentes acerca de la clasificación pampa/ch'uru. Por un lado, ratifican lo enunciado en investigaciones anteriores (cf. Arnold y Espejo 2007; Cereceda 2010; Fisher 2011; Gisbert et al. 1987; Medvinsky 1977; Silvermann 2002; Torrico 1989; Zorn 1987); por el otro, agregan datos sobre los campos semánticos asociados a la distinción cristiano/no cristiano y así, con los antepasados.

Presentamos, en primer lugar, el contexto social en el que se producen las fajas, su composición y su vinculación con las prácticas sociales. Continuamos con una descripción de los diferentes tipos según los criterios relevados por las tejedoras. Así, se identificaron los principales componentes del diseño y sus significados asociados de las fajas pampa y de las fajas ch'uru. Indagando en las bases de esta clasificación se observan elementos vinculados con la muerte, con los carnavales (antepasados) y con las divinidades cristianas. A modo de ilustración, exponemos las "lecturas" de las tejedoras de dos casos de fajas pampa: Wak'a Kili y Chilenito Pampa. Para conocer la composición de las fajas ch'uru, describimos las fajas ch'uru chilenito, carnerito simple, carnerito triple y las fajas pusi ch'uru. En la tercera parte ofrecemos antecedentes que permiten avanzar hacia una interpretación de los conceptos y que nos remiten a dominios semánticos-seculares y religiosos-inscritos en los procesos de dominación colonial.

\section{Los Diseños y su Vinculación con los Seres Tutelares}

La palabra en lengua aymara para designar estas piezas es wak'a. Se trata de una cinta de $4-5 \mathrm{~cm}$ de ancho por $140 \mathrm{~cm}$ de largo, aproximadamente. $\mathrm{Su}$ función principal es ajustar en la cintura el vestido de mujeres y hombres, niños y adultos.
Hasta la década de 1980, en Isluga y Cariquima las jóvenes regalaban fajas a sus pretendientes para manifestarles su afecto y su interés por ellos. Esto se relacionaba con el hecho de que la actividad textil formaba parte de las labores femeninas y, por tanto, constituía un indicador de su desempeño como futuras esposas. Las habilidades textiles se evidenciaban en la festividad de carnavales. Sus trajes eran especialmente observados por los hombres casamenteros y sus familias. Una mujer que usaba el mismo vestido del año anterior era calificada como mujer floja. De igual manera lo era aquella que osaba llevar un vestido mal tejido (Gavilán y Ulloa 1992).

En estas comunidades se registran los siguientes tipos de fajas: k'ili wak'a, chilenito wak'a, ch'uru chilenito wak'a, carnerito simple, carnerito doble, carnerito triple, kimsa ch'uru, pusi ch'uru, saldani wak'a (esta última incluye varias modalidades identificadas por el número de pares de hilos o chinu). Se pueden identificar variantes de estas piezas, las que se nombran según sus diseños particulares.

La circunstancia social definía el tipo de fajas adecuada para vestir y lucir. Eran tan importantes en la vestimenta diaria que las mujeres recuerdan haber tejido todos los meses para abastecer a sus parientes y para su propio uso. Aunque en las décadas de los setenta y ochenta el traje tradicional comenzaba a ser reemplazado por vestidos manufacturados industrialmente, se siguió utilizando durante las festividades. Aún así, las personas mayores las llevan debajo de la ropa, ajustadas en la cintura, para aliviar malestares en la columna. Las entrevistas indican una clara distinción entre el vestido cotidiano y el festivo:

Para las fiestas se hace puro madeja, sin alpaco. Hay que buscar el color, según cuál sea más bonito. En las fiestas las k'ili se ponen abajo, encima se ponen las carnero. A mí me gusta ponerme encima las fajas con saldas.

Los diseños no han quedado estancados en el tiempo. Se han ido transformando.

Yo hacía fajas más largas, no hay fajas más anchas, quedan muy feas. Pero antes había fajas antiguas más anchas, payao acá, payao allá, como nueve pares había que poner al medio. Antiguo es eso. 
Esta faja [fajas con salta] es más difícil de tejer que las otras. Estas llegaron después que las carneritos.

.... mí, mi abuelita me contó que la gente de Cariquima antes tejían más gruesito y las de Isluga más finito. Las de Cariquima tenían k'isa, los de Isluga no tenían k'isa. Los aksos de las abuelas eran negros o café entero y en las orillas ponían verde, azul, así. Después los Isluga copiaron $k$ 'isa para todo.

Los tejidos de Cariquima antes combinaba más bonito, pero siempre las k'isa resaltaban.

Yo conocí a mi abuelita con akso hasta abajo de color negro o chumpi, en Isluga es caído mismo, hasta el tobillo, ancho y plisado (regular no más). Después se empezó a subir de a poco. Primero empezó en Cariquima. Después copiaron en Isluga.

Hay fajas para días de trabajo, fajas para calmar malestares del cuerpo, fajas para los bebés recién nacidos, para carnavales y para vestir los cuerpos sin vida:

Cuando se fallecen las personas se le pone la faja y akso que más le gustaba a la finada, las demás se queman.

Cuando la gente se fallece se pone $k^{\prime} i l i$. El carnero no se puede poner, esto no se puede leer. Con el k'ili se va derechito al cielo, otro no pu está partido. Dios se enoja. Castiga decía mi abuelita, esta figura no se ponía a Dios. Pampa chilenito también se puede poner. El k'ili o palma por castellano, suerte sabe decir, trae suerte. Pal viaje se ponía. Mi abuelito de dos o tres, hasta cinco se ponía para amarrar los pantalones de jerga, como cinturón, se gastaba mucho, cada mes hay que tejer.

Cada tipo de faja está asociada a momentos y estos están relacionados con el calendario ritual, con los antepasados y divinidades. Hallamos, por ejemplo, una asociación entre las fajas carnerito con las fiestas de carnavales, celebración del encuentro con los antepasados. Todos los diseños ch'uru son apropiados.

Los diseños definen los nombres de las fajas y su uso. Las fajas talbilla o tablilla eran usadas por las abuelas. Las fajas kili son identificadas como antiguas y eran las que más se utilizaban. Wilma, nos informa que:
A las abuelas no les gustaban las fajas que tejen ahora, por ejemplo kimsa ch'uru no les gustaba. Mis abuelos tejían con caito finito hilado y después teñíamos. Mi abuelita teñía, después torcía, después tejía. Después llegó madeja chilena de tres o cuatro hebras. Los abuelos de Chiapa hasta ahora usan estas fajas, en Mauque también usaban de dos o de tres.

Desde el punto de vista de su función las tejedoras distinguen dos grupos de fajas: (a) pampa y (b) ch'uru. Aquellas piezas que se estructuran en pampa, son permitidas por las divinidades cristianas; en tanto que las fajas ch'uru representan rupturas que, según las mujeres, el mundo cristiano rechaza:

cuando se muere la gente no puedes poner carnerito, ¡qué va a decir el dios, qué está diciendo esta gente dirá!

De este modo, los diseños pampa forman parte del ajuar mortuorio y para la realización del "viaje" que emprenderán y los vinculará con el Dios cristiano. En tanto que las fajas ch'uru son apropiadas para el vestido de los carnavales. Para describir el proceso de elaboración, las tejedoras indican: "uno está botado otro está andando"; es decir mientras los hilos de color claro están por arriba, los oscuros se mantienen por su revés. Cada una de ellas se compone en dos partes y un centro, siguiendo una estructura simétrica. Los segmentos se elaboran a base de tres criterios: hilado-torcido (en $\mathrm{Z}$ o S); color (saturados o en gradaciones) y número de chinu (pares de nudos o pares de cruces de hilos) ${ }^{1}$. El centro (taypi o chimma) define tanto el tipo de faja y, por tanto, su nombre específico. Veamos un ejemplo:

Segmentos de las fajas k'ili (corte transversal del tejido) (Figura 1).

En este caso el hilado de las partes siempre es $\mathrm{S}$ y el torcido es Z. El color rojo define las orillas denominadas vila lak'a (boca roja). El color plano y las degradaciones ( $k$ 'isa) configuran las partes; en tanto que la figura central da el nombre a la faja ( $k^{\prime}$ ili wak'a) y la clasifica en el tipo pampa. 


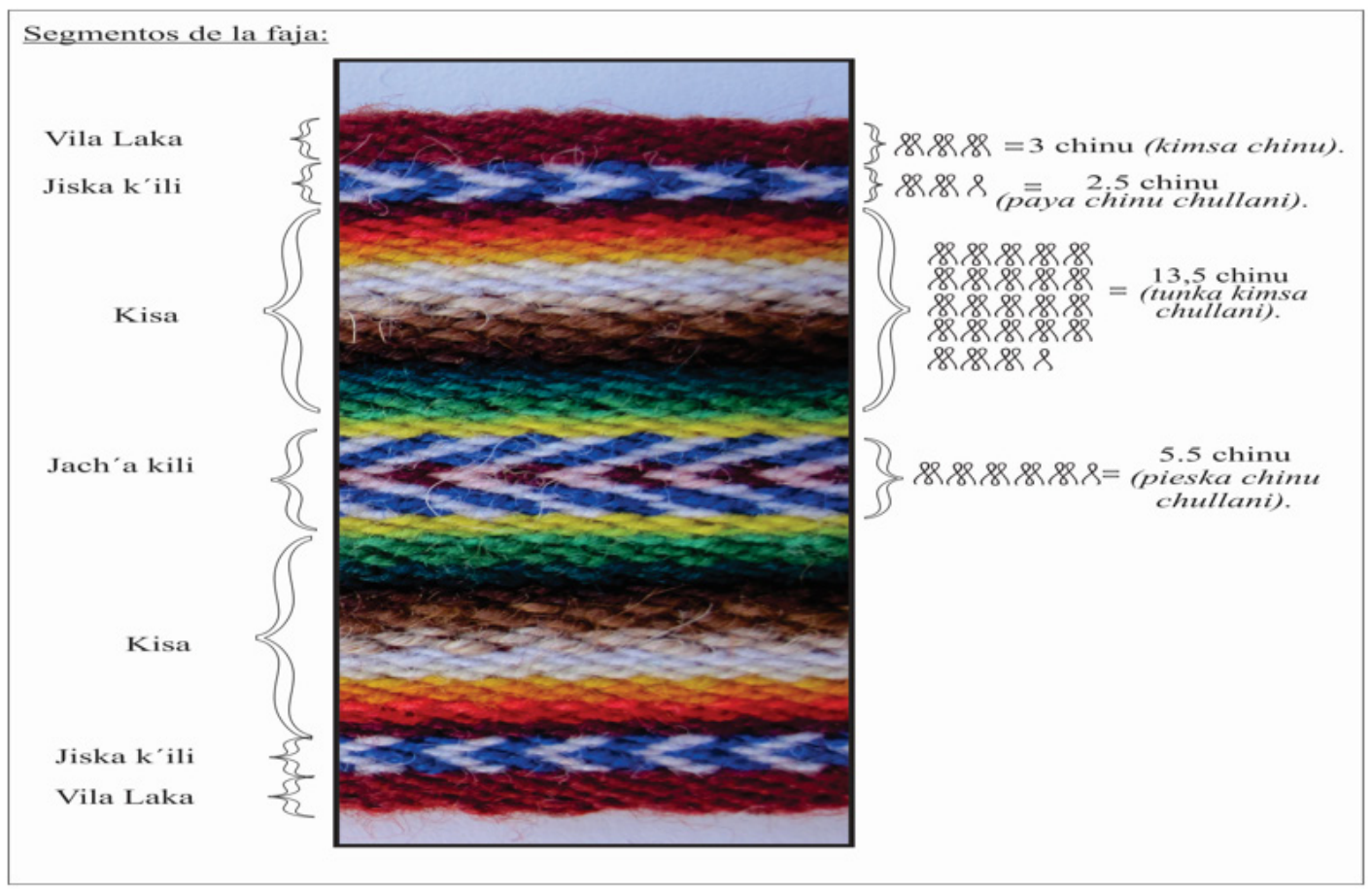

Figura 1. Segmentos de las fajas k'ili (corte transversal del tejido).

Segments of the k'ili belt (cross-section of the textile).

\section{La Lectura de las Tejedoras de las Fajas Pampa}

Faja K'ili (Figura 2)

\section{Urdido}

Lak'a o boca. Puede ponerse rojo o guindo. Se compone de tres chinu o tres cruces de hilos.

Jiska K'́li ( $K^{\prime}$ ili chico). Esta figura tiene dos y medio chinu (paya chinu chullani): uno de color azul, uno y medio (maya chullani) de color blanco.

Pampa. Siempre lleva k'isa. Está compuesta por tres k'isas: Jaruma k'isa o k'isa de color naranja, café $k^{\prime} i s a$ o $k^{\prime}$ isa de color café y chojña k'isa o k'isa de color verde. En total son tunka kimsa chullani o trece pares y medio.

J'acha k'ili (k'ili grande). Se urdieron cuatro chinu un chinu de color blanco, un chinu de color azul, un chinu guindo y un chinu rosado.

Pampa: se empieza con el color que se terminó el otro lado de la pampa.

\section{Pampa Chilenito Wak'a}

Se define por su tejido plano, sin cortes en el centro. La figura central es la que le da el nombre de chilenito. Algunas mujeres la llamaron nayra (ojo). Le sigue chulla linkhu nayraja, chilenito, nayra/chulla nayra, nayra completo.

\section{Pampa Chilenito Wak'a (Figura 3) Urdido}

Lak'a. Está compuesta por tres chinu k'isados (gradaciones): uno de color blanco, uno de color k'ose o beige, uno de color café. En este caso tiene k'isa en la boca. La tejedora que "leyó" esta pieza dice: "seguramente, la señora que tejió no tenía lana de color rojo o guindo. Si tenemos ponemos, si no, no".

Linkhu. Se compone de dos chinu de color rosado; ocho chinu de color blanco; dos chinu de color café. En total hacen doce chinu o doce pares de hilos. 


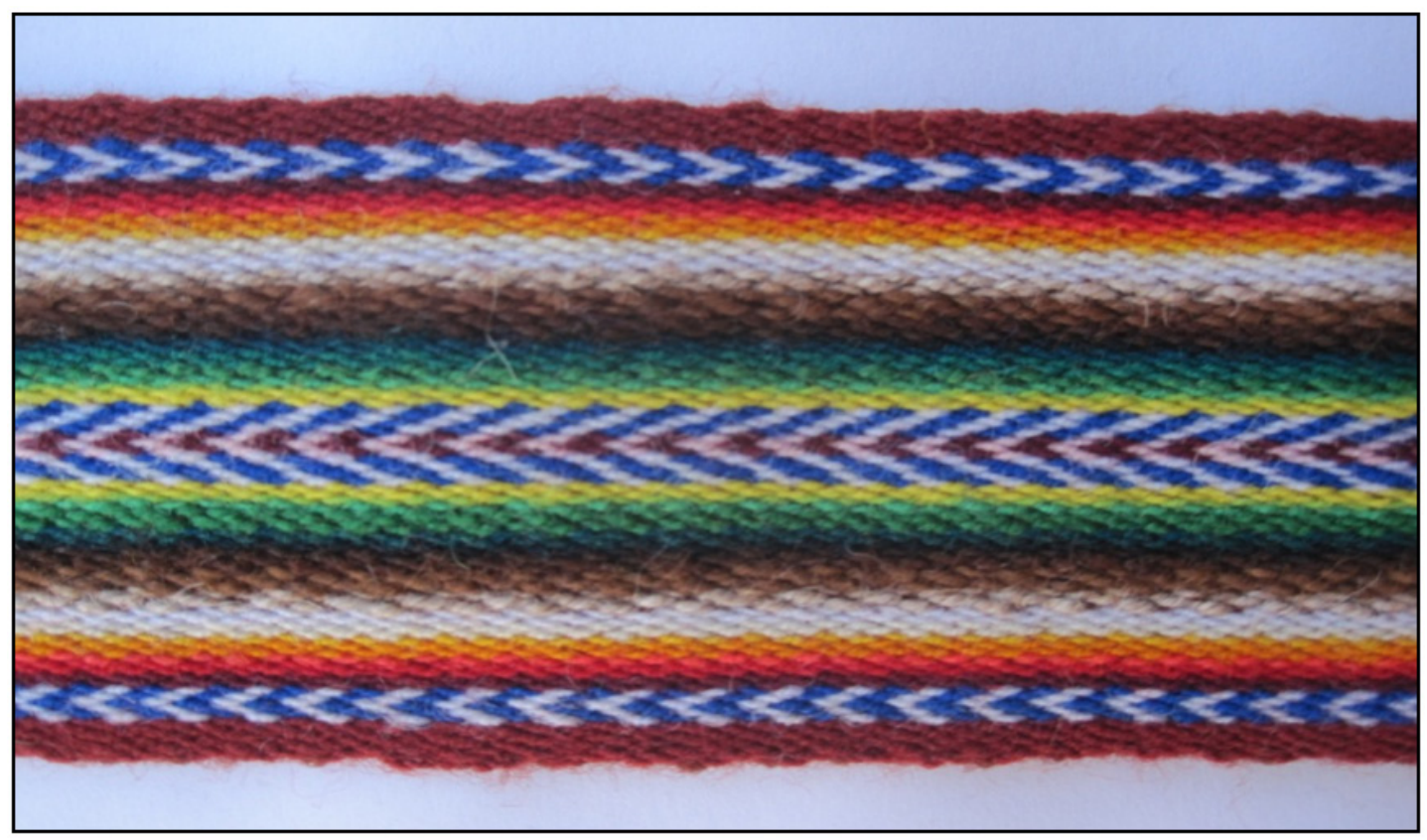

Figura 2. Faja Pampa K'ili Wak'a.

Pampa K'ili Wak'a belt.

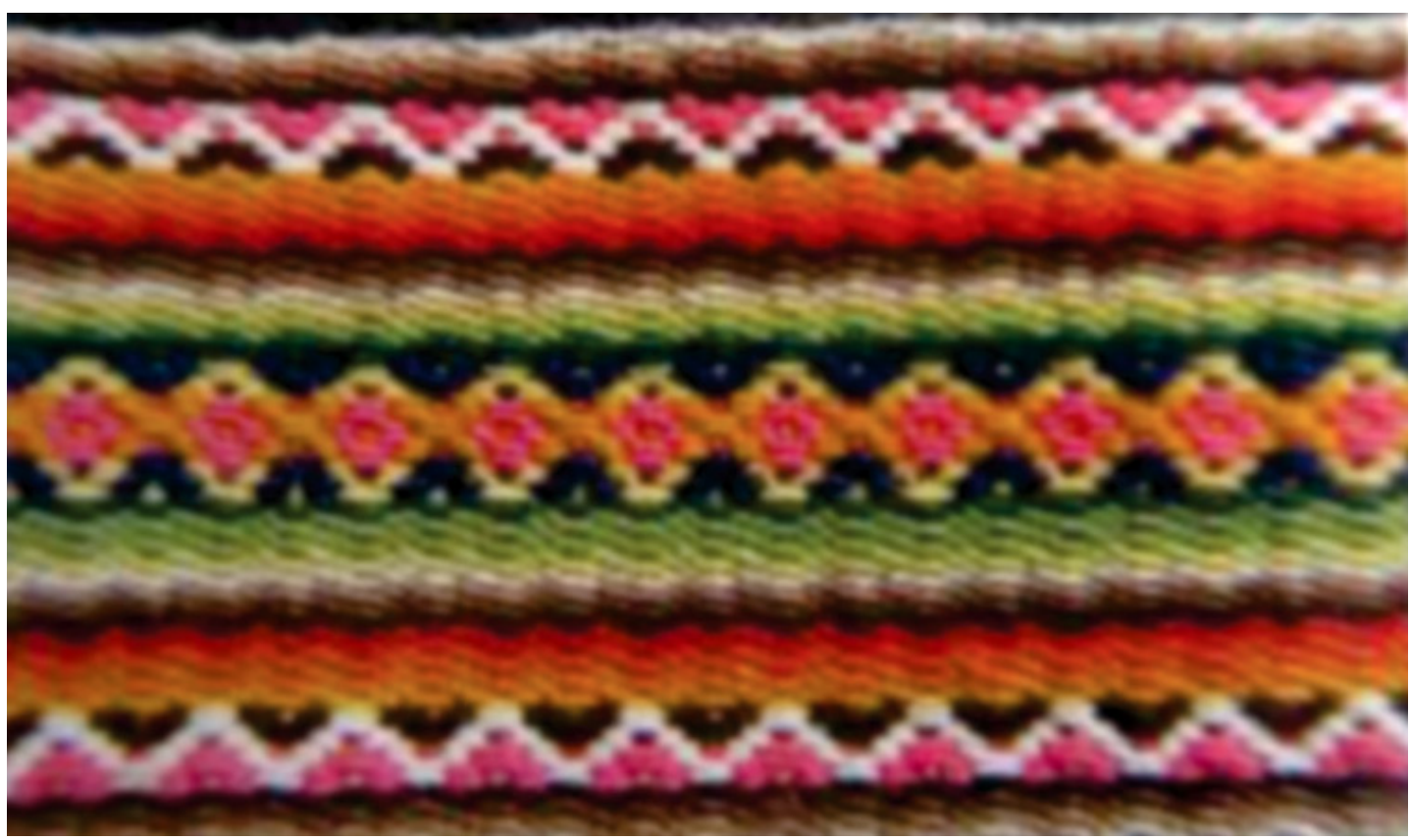

Figura 3. Pampa Chilenito Wak'a.

Pampa Chilenito Wak'a belt 
K'isa. La pampa kisada se compone de un chinu de color amarillo; un chinu de color naranjo; un chinu de color rojo; un chinu de color k'ose; un chinu de color blanco; un chinu de color amarillo; un chinu de color verde loro; dos chinu de color verde; un chinu de color verde oscuro.

Taypi o centro. Chilenito o nayra: cuatro chinu de color azul; seis chinu de color rosado; dos chinu de color amarillo; cuatro chinu de color amarillo más fuerte. En total son 16 pares.

Se repite igual.

\section{La Lectura de las Tejedoras de las Fajas Ch'uru}

\section{Faja Chilenito Ch'uru (Figura 4) Urdido}

Lak'a. Vila lakani compuesta por tres chinu.

Linkhu. Cuatro chinu. Uno rosado, dos blancos y uno negro.
Pampa. Compuesta por una $k^{\prime} i s a$ de color azul (laram k'isa) y otra de color naranja (jaruma k'isa; Once chinu (tunka mayani) en total; dos chinu guindo con hank'o, dos chinu vila con hank'o larama, dos chinu jaruma vila con larama, dos chinu jaruma con larama, dos chinu kellu con larama, un chinu hank'o kellu con chara morado.

Taypi o centro compuesto por chilenito nayraya con hilos de color rojo. Esta figura fue nombrada también como pachanayra o ojos del universo. Se dispuso siete chinu (pakalku chinu) de color blanco, verde y rosado.

Se repite la pampa que es nombrada como $K^{\prime}$ isa ch'uru o k'isa del ch'uru.

\section{Faja Carnero Simple (Figura 5) Urdido}

Lak'a o boca. Compuesta por tres chinu de color rojo.

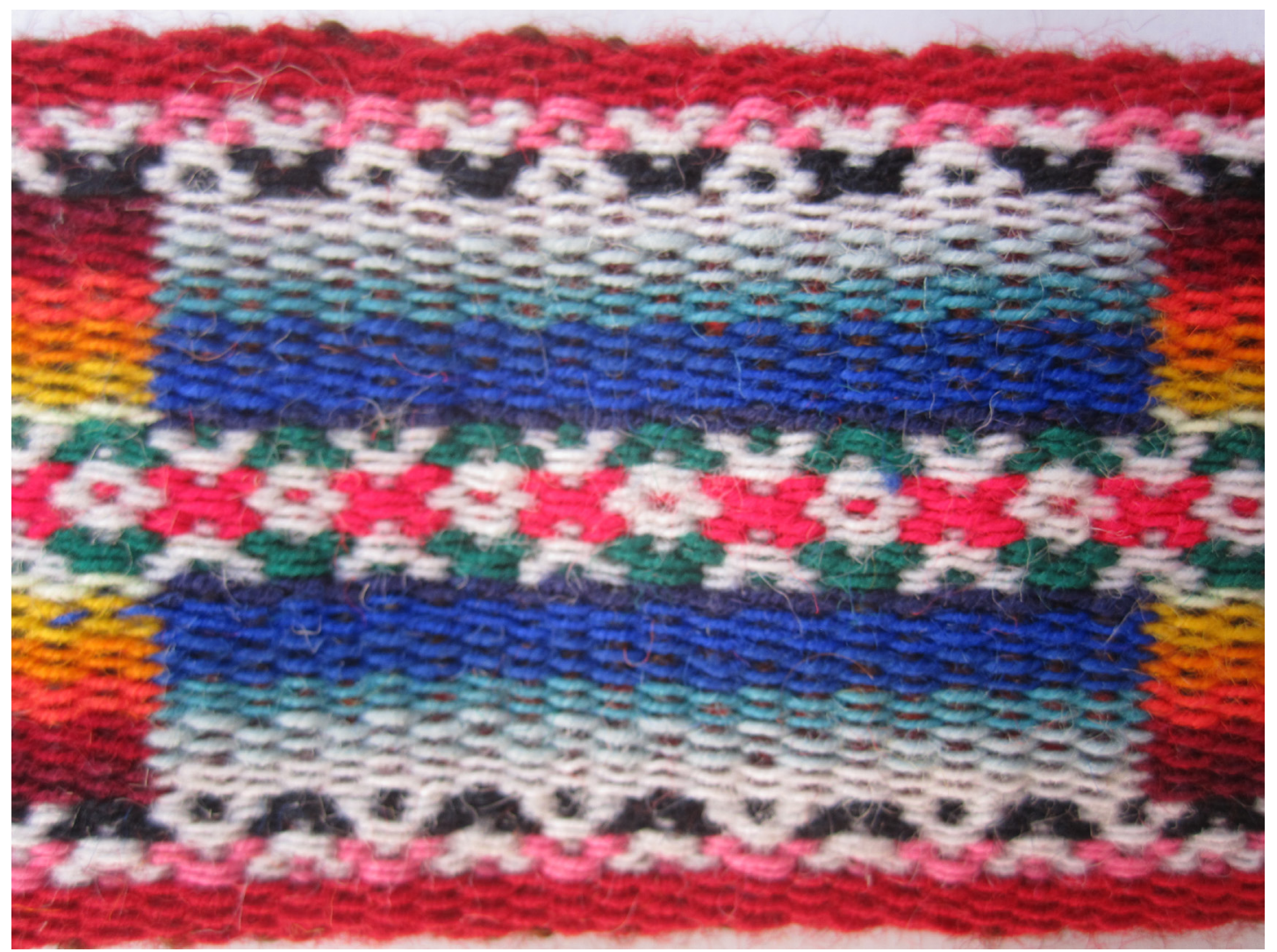

Figura 4. Faja Tipo Ch'uru: Ch'uru Chilenito Wak'a.

Ch'uru type belt: Ch'uru Chilenito Wak'a. 
Linkhu. se compone de seis chinu y medio (sojta chinu chullani). Dos chinu de color blanco, dos chinu de color naranja, dos chinu de color azul y medio chino de color negro. Chojña K'isa (k'isa de color verde). Se configuró con tres chinu y medio (kimsa chinu chullani). Medio chinu o chulla chinu de cada color. Ch'uru: se compone de 19 chinu de color negro y 19 de color blanco, para elaborar la figura que representa a Suri Kullalla. Continúa con tres y medio chinu (kimsa chinu chullani) para formar la k'isa de color rosado o lirio k'isa.

Se repite la misma estructura, tres y medio chinu (kimsa chinu chullani), pero cambia de color. Se escogió lirio k'isa o bandas de color rosa en degradación.

\section{Ideogramas}

Las figuras que contiene cada ch'uru aluden a flores, aves, llamas y alpacas y algunas imágenes que las tejedoras aprendieron de sus madres o abuelas, recuerdan sus nombres pero no sus significados.

\section{Faja Carnerito Triple (Figura 6) Urdido}

Lak'a. Vila lakani (boca formada por hilos de color rojo) compuesta por tres chinu.

Linkhu. Dos chinu de color naranja, ocho chinu de color blanco y dos chinu negros. Medio chinu verde (chinu chullani) marca el ch'uru.

Ch'uru. 16 chinu blancos (tunka sojtani); 16 chinu de color lila. Para marcar el ch'uru dispone de medio chinu rosado (chinu chullani), 16 chinu rojos, 16 chinu de color verde, 16 chinu verde loro, 16 chinu guindo; medio chinu verde para marcar el tercer ch'uru.

\section{Faja Pusi Ch'uru Wak'a o Cuatro Ch'uru (Figura 7) Urdido}

Lak'a. Compuesta por tres chinos de color rojo: Vila lakani.

Linkhu. Cuatro o pusi chinu. Un chinu de color anaranjado o jaruma, dos chinu de color blanco o hank'o y un chinu de color azul o larama.

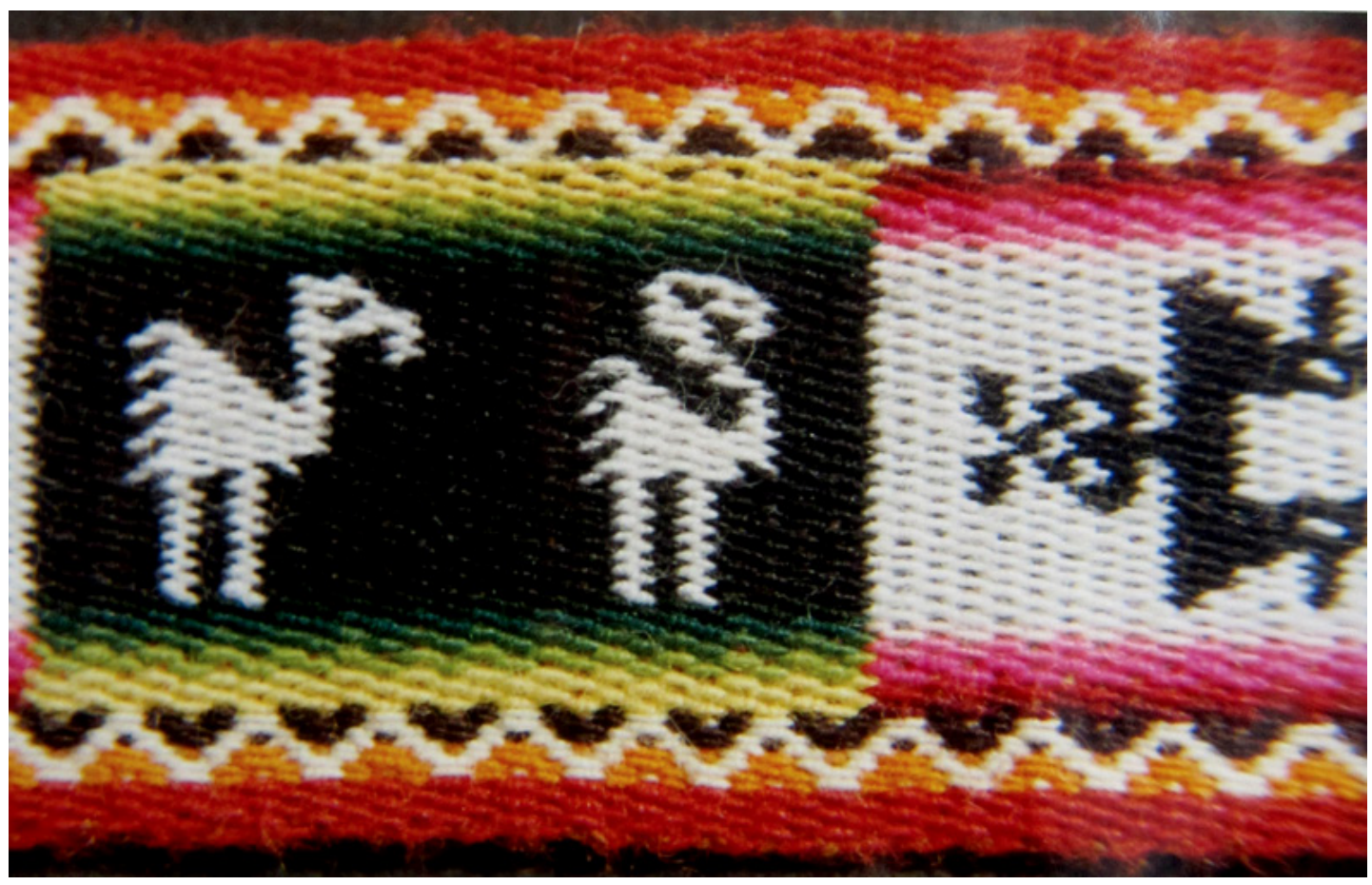

Figura 5. Faja Tipo ch’uru. Chulla Carnerito representación de Suri Abuela o Suri Kullalla, personaje mitológico. Ch'uru type belt. Chulla Carnerito (little ram) representation of Suri Abuela or Suri Kullalla, a mythological character. 


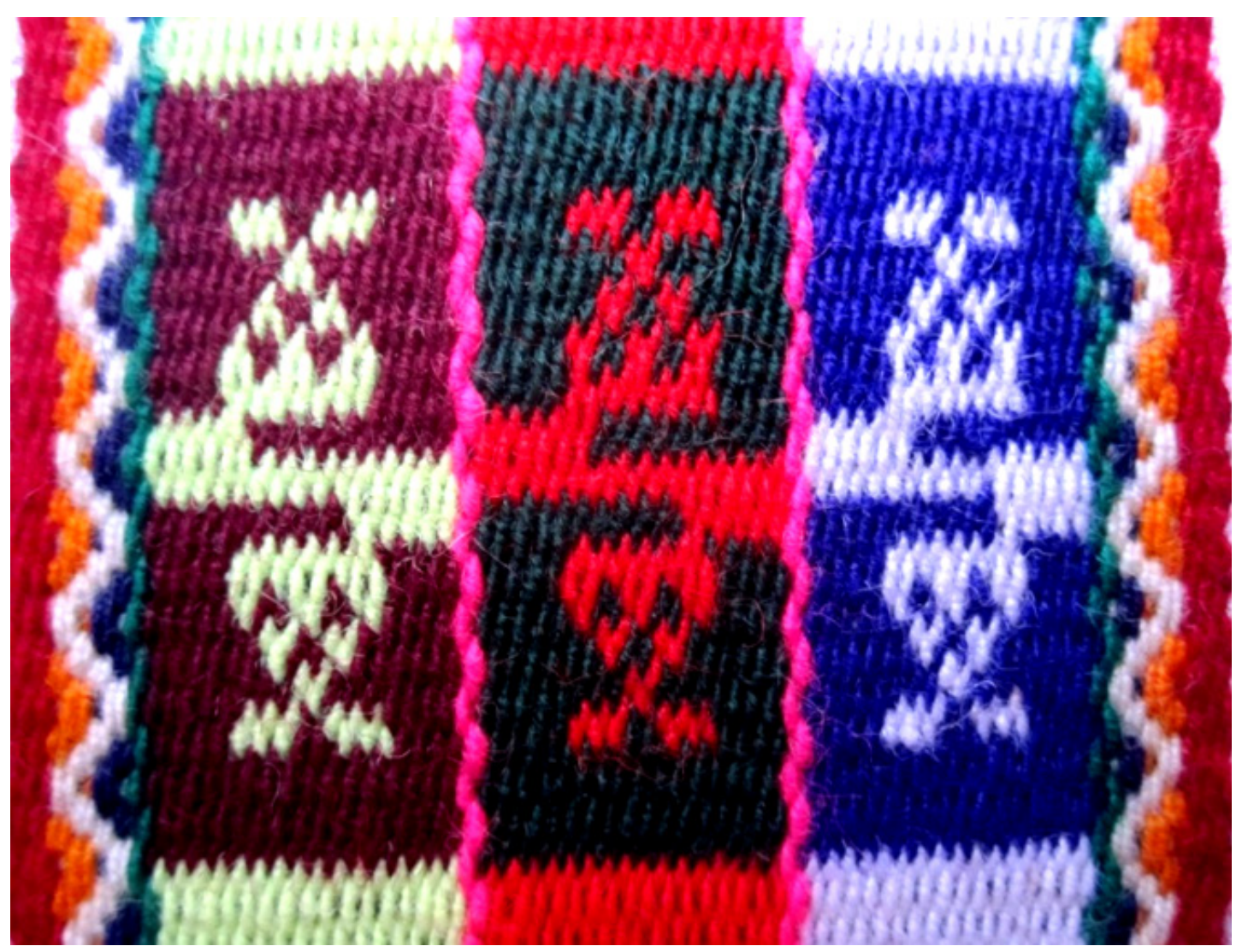

Figura 6. Faja Tipo ch'uru. Carnerito triple con la imagen de un T'ok'o búho, anuncia un brujo.

Ch'uru type belt. Triple Carnerito (little ram) with the image of a T'ok'o búho, anouncing a shaman.

$K^{\prime}$ isa. Se compuso con seis pares de hilos o chinu de color verde: chojña k'isa, seis de color rosado o lirio k'isa.

Taypi: Ch'uru Carnero formado por 14 pares de hilos. Tres pares de hilos y un impar de color verde o chojña, la misma cantidad para el guindo, para el color lirio o rosado y para el blanco. De este modo, cada color compone a un ch'uru o cajón, dando como total cuatro ch'uru o pusi ch'uru.

Las figuras que contiene cada ch'uru son similares a cualquier faja carnerito.

\section{Los Conceptos de Pampa/Ch'uru}

La lectura de las tejedoras señalan que las fajas ch'uru son nominadas también como fajas con warsu o fajas con khuchu. Términos que aluden a espacios recortados. La palabra ch'uru en el diccionario de Bertonio (1984) indica pedazo de tierra u otras cosas como paño; o pedazo de chacra. Lo mismo significa en las comunidades rurales de la región
Arica y Parinacota. Un ch'huru para cada uno de los hijos ${ }^{2}$, por ejemplo. En contraposición, pampa se refiere a planicie o espacio sin interrupción. En Bertonio encontramos, también, la palabra warsu warantaña, que se refiere a "derramar y volver a echar muchas veces". Por su parte khuchu alude a cortar, kuchunuqata pedazo de paño cortado.

La clasificación pampa/ch'uru es descrita por Medvinsky (1977:59-61). Señala que en Isluga "las fajas chilenito sin ch'uru, se caracterizan por llevar una $k^{\prime} i s a$, sin ser cortada a lo largo de la faja, en su franja central no varían en color ni forma. Las fajas chilenito con ch'uru, en cambio, lleva la k'isa cortada, produciéndose espacios cerrados, llamados ch'urus, y aparecen en cada uno de ellos en colores diferentes que se van alternando".

Agrega que las fajas carnero hacen referencia iconográficamente a motivos de flora y fauna del lugar. Se pueden subdividir en fajas carneros y fajas carnero kimsa ch'uru. Las primeras se subdividen en fajas carnero simple, que se caracterizan por 

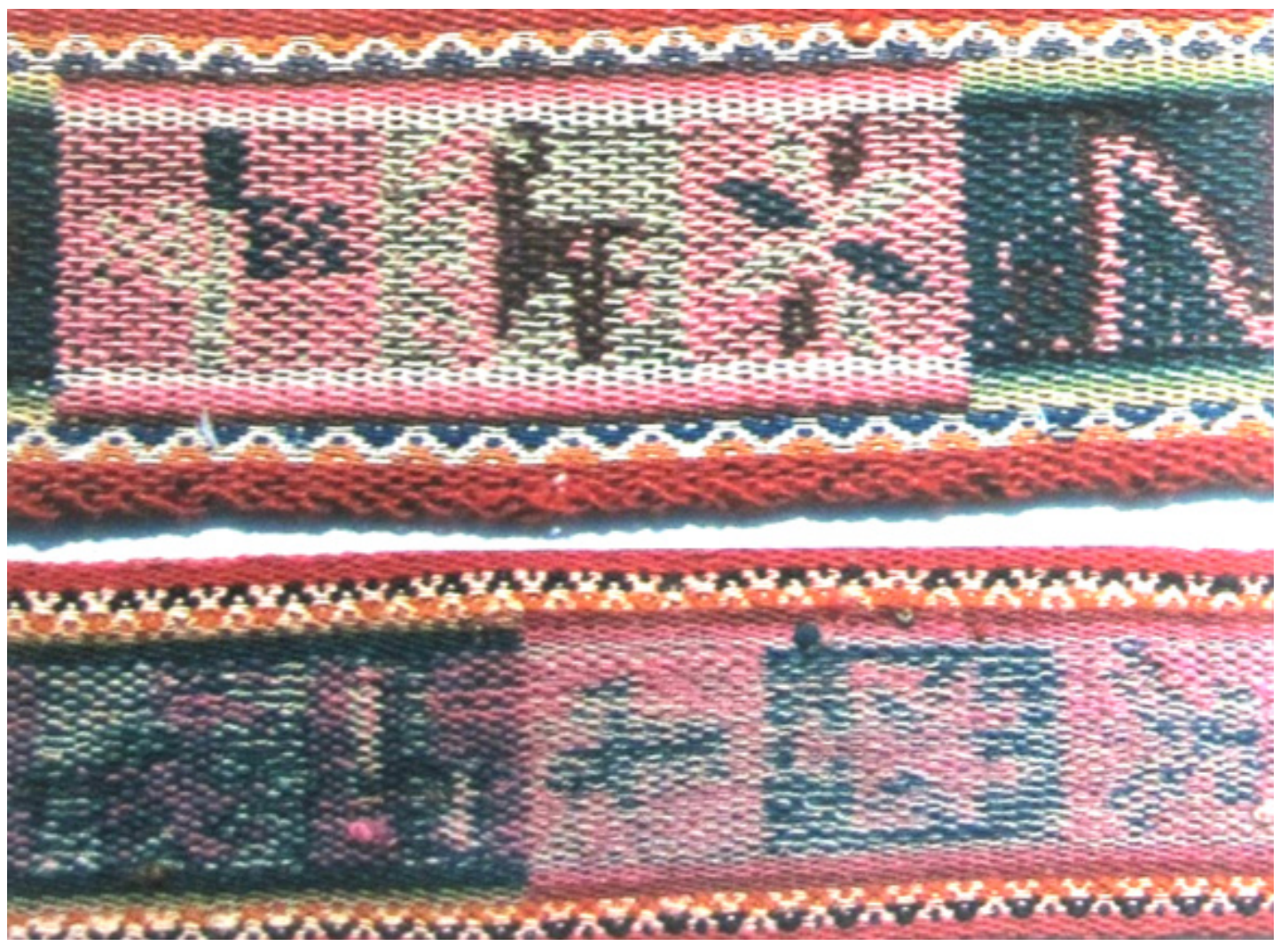

Figura 7. Faja Tipo ch'uru. Ch'uru Wak'a o Cuatro Ch'uru.

Ch'uru type belt. Ch'uru Wak'a or Four Ch'uru.

llevar dibujo en una franja central, distribuidos en espacios cerrados. O fajas carnero doble que se diferencian porque estas llevan dos cuerdas de figura paralelas, siendo divididas por una franja central que es más angosta. En este caso el ch'uru es muy angosto, el color puede variar en ambos lados y puede ser reemplazado por una línea, caracterizada por lo general de color rojo. Medvinsky (1977) se refiere a lo que las tejedoras llaman un chinu telaja para marcar los ch'uru. Afirma que la ubicación de ch'uru y sus variaciones son dos. La primera es que el ch'uru pueda abarcar un fondo que encierre una figura, y el segundo puede llegar abarcar dos fondos distintos, con motivos diferentes. Las fajas kimsa ch'uru presentan las mismas características formales de las fajas carnero anotadas anteriormente. Solo se diferencian en su aspecto técnico. Presentan los motivos en tres colores distintos a la vez (Medvinsky 1977:75).

Este estudio confirma lo expuesto por esta autora y agrega otras observaciones. Es posible indicar que los diseños se pueden ordenar por tipo en: k'ili, chilenito, carnerito. Cada uno de los cuales se distinguen, a su vez, en ch'uru o pampa. Hallamos que las fajas carnero siempre son ch'uru y pueden ser; simple, doble o triple, si van en una sola banda, en dos o tres, respectivamente, a lo largo de la faja. Asimismo, de acuerdo con el número de ch'uru, pueden ser kimsa (tres) o pusi (cuatro) o trazos de colores alternados en claro/oscuro/claro.

Cereceda $(2010 \text { [1977]) })^{3}$ coincide con Medvinsky. Sugiere que ch'uru es el término que dan las tejedoras a las bandas dispuestas en las talegas. Lo cual también puede ser nominada como tayka. Para ella, en su aspecto formal, ch'uru; o bandas, manifestarían las siguientes ideas fundamentales: ch'uru sería "cajón", en los que se puede guardar algo, depositar algo. Expresaría, de igual modo, la idea de protección. Observa además que los ch'urus serían siempre estrechos y encerrados. Y por medio de esa característica se relacionan no solo con habitación o receptáculo, sino con surco o camellón 
agrícola. De esta manera, la primera propiedad de los ch'urus es determinar espacios angostos, cercados, que encierran, guardan o protegen, como un cajón, un surco o una casa redonda.

En el lenguaje textil, afirma, el ch'uru tiene su opuesto: la pampa. Si en el ámbito topográfico pampa designa una planicie extensa, en el tejido, pampa es un espacio amplio, de color uniforme y sin interrupciones. Así, pampa es abierta / ch'uru es cerrado. Pero también, más allá del tejido, pampa es natural / ch'uru es cultural. Oposiciones que -organizándose con otros pares como continuo/discontinuo, natural/ teñido, ancho/estrecho, etc.- conforman el sistema que subyace tras la manifestación del diseño. En tercer lugar, los ch'urus se presentan siempre en sucesión. Afirma que no es posible encontrar en los textiles de Isluga un espacio uniforme recortado de pronto por una banda solitaria de otro color. Los ch'urus -al inscribirse en un espacio- lo transforman en discontinuo, determinando siempre una superficie subdividida, labrada, llena. Y se oponen nuevamente, por este aspecto colectivo, al concepto de pampa mencionado más arriba. Ch'uru es discontinuo/pampa es continuo, ch'uru es colectivo/pampa es singular.

Gisbert (1988:16) sigue estas ideas, argumentando que pampa tiene la misma acepción que en su contexto geográfico, asociado al mundo salvaje -no cultural- semidesértico. Señala que en el campo textil, la pampa designa el sector uniforme, carente de decoración, en contraposición del pallai (sección que combina hilos de colores para representar figuras) que es la parte decorada. Señala que esta parte representa el mundo cultural con significado; contiene representaciones de los dioses y linajes y allí también estaría el mundo circundante.

Zorn (1985), por su parte, se refiere a las k'uchu 'unkhuña en Macusani como las mantas pequeñas cuatripartitas ( $\left.k^{\prime} u c h u\right)$ que se utilizaban como altar (misa) o bien para recibir los pedacitos de oreja cortados a los animales marcados. Así, indica que cuando el campo central de las mantas, divididas en dos mitades, siguiendo el eje vertical de la urdimbre es vuelto a dividir siguiendo el eje horizontal de la trama, se forman cuatro cuadrados de cuatro colores distintos. La autora busca definiciones del término khuchu e informa que "kocchu se refiere a esquina de las piedras, paredes y rincón de la casa. El anónimo (1614) dice Cuchu, cantón, esquina, cosa quadrada" (Zorn 1987:520).

Como se ha mencionado antes, la clasificación elaborada por las tejedoras de Isluga y Cariquima distingue dos grandes tipos de fajas: fajas pampa y fajas ch'uru ${ }^{4}$, diseñadas para el uso según los contextos cotidianos o festivos y estos asociados con el mundo cristiano/no cristiano (ver supra). La pregunta lógica es ¿por qué los diseños pampa se asocian al mundo cristiano?, y ¿por qué los diseños ch'uru se relacionan con los antepasados (aquellos no cristianos)?

Si bien los hilos de las fajas tienen dirección hacia la derecha y torcidos a la izquierda, algunos de ellos, o partes de un mismo tejido, puede seguir la dirección contraria. Las tejedoras asocian el hilado a la izquierda con los difuntos (y demonios) y la lucha entre la izquierda y la derecha es parte de las sanaciones de las enfermedades provocadas por estos. Por ejemplo, el yatiri o sanador realiza un acto de intercambio o turka con las divinidades/demonios/ antepasados, para recuperar el animu del enfermo mediante el sacrificio de un animal, acompañado de un hilo blanco y un hilo negro que se tuerce a la izquierda (ch'ika). Estos hilados sirven también para marcar los corderos y llamos, y para curar a la gente. Se pasa en todo el cuerpo. Encontramos también algunas llijllas cuyas orillas son torcidas a la izquierda ch'ika laka y estas son las uywa kepe, que guarda todos los elementos rituales del wayño (Titi, Chullumpi, sogas, ticas, etc.). Es especial y solo se utiliza con fines rituales.

La disposición de las fajas pampa en la vestimenta de las personas fallecidas se justifican por la asociación del tejido llano, sin recortes y sin representaciones de animales y otras figuras con el Dios cristiano y de aquí con el cielo.

Si seguimos la interpretación de Cereceda (2010 [1977]) y agregamos las asociaciones que realizaron las tejedoras vemos lo siguiente:

\begin{tabular}{ll}
\hline \multicolumn{1}{c}{ Pampa } & \multicolumn{1}{c}{ Ch'uru } \\
\hline Espacio abierto & $\begin{array}{l}\text { Cajones que protegen, } \\
\text { encierran } \\
\text { Receptáculo/continente } \\
\text { Planicie }\end{array}$ \\
Tierra sin límites & $\begin{array}{l}\text { Surco o camellón agrícola } \\
\text { Ciscontinuidad }\end{array}$ \\
Continuidad & Colectivo, plural \\
Solitario, singular & Cultural \\
Natural & $\begin{array}{l}\text { Fajas para vestir en la fiesta de } \\
\text { carnavales }\end{array}$ \\
Fajas para vestir a los & Hilado a la izquierda \\
difuntos & Mundo de los antepasados \\
Hilado a la derecha & (Demonios/Manqha Pacha) \\
Mundo cristiano (Dios/ & \\
Cielo) &
\end{tabular}


Las expresiones de las tejedoras para explicarnos las funciones de las fajas: "el carnero no se puede poner cuando se fallece", "esto no se puede leer" o "con el $k$ 'ili se va derechito al cielo, otro no pu está partido, Dios se enoja, castiga decía mi abuelita, esta figura no se ponía a Dios"; son señales de la distinción que realizan las familias aymara entre las divinidades coloniales y las del pasado indígena. El concepto de pampa, como espacio llano, sin interrupciones y como "no cultivado" es asociado al cielo como referente del Dios cristiano. Así, pareciera ubicarse en el desorden comprendido como espacio en el que no se observan las normas de convivencia legadas por los antepasados, sino aquellas impuestas por los q'ara. En tanto que ch'uru, como continente y los ideogramas como contenido, hablan de la vida en una matriz que protege; protección que se pide a los antepasados asociado al mundo demoniaco, como efecto de los procesos de dominación.

La referencia del centro de las fajas tipo chilenito o nayra pacha nos remite a la entidad totalizadora (espacio-tiempo), que resume una deidad tutelar que propicia la vida pensada antropomórficamente. La naturaleza viva del textil es sostenida también por Zorn (1987) y Torrico (1989). Arnold (2007:55), apoyada en estas interpretaciones, habla también de la corporalidad textil que no solo semantiza las relaciones generativas entre una madre y sus crías sino también entre los caseríos y las comunidades ubicadas a distintas alturas. De este modo, el concepto de ch'uru pareciera aludir a continente o espacio uterino, un lugar de protección y su vinculación a las divinidades del pasado, a lo oscuro y demoníaco; pero al lugar de abajo, allí donde está la vida.

Solo una tejedora experimentada logra una pieza estética y funcionalmente correcta, puesto que la composición de las fajas ch'uru se orienta a la obtención de una iconografía compleja que busca conectarse con los antepasados en un lenguaje que comunica la idea de lo que debe ser y lo que la memoria indica para la vida social.

\section{Reflexiones Finales}

La memoria colectiva promueve la continuidad del culto a divinidades ancestrales. El pasado sigue en el presente con las transformaciones que han impuesto los procesos de dominación colonial. A pesar de la evangelización, la presencia de seres tutelares del antiguo mapa religioso emergen en los intersticios de las prácticas sociales. La apropiación de la ideología cristiana por parte de los y las comunero/ as incluye conceptos que no pueden ser reemplazados para pensar la vida y estar en el mundo. Estas ideas abstractas se plasman en soportes materiales como la textilería. El hilado y su torsión, los colores y la contabilidad (en pares o chinu), constituyen las bases de un lenguaje formal abstracto que fue y es elaborado para comunicar, para registrar y memorizar, pero que todavía resta por comprender.

Los conceptos ch'uru/pampa incluyen referencias a campos semánticos que pueden contribuir a confirmar ideas relacionadas con la fertilidad (productividad) y la reproducción biológica y social, con un tipo de filosofía que distingue lo cultural de lo natural a partir de un sistema normativo legado de los ancestros, de los seres tutelares que promueven el bienestar del espacio-tiempo y la continuidad de la colectividad.

Los resultados del estudio de las fajas tienden a confirmar los datos de otras investigadoras (Cereceda 2010; Gisbert 1887; Medvinski 1977; entre otras). El punto para el debate es que estos antecedentes indicarían que la idea de ch'uru en las comunidades referenciadas se asocia al mundo de los demonios o saqra, espacio que ha tendido a calificarse como caótico y salvaje. Pero en la expresión textil, justamente el espacio cultivado y de contención de los carnavales y/o antepasados se ubican en el orden de lo social. En tanto que pampa se vincula directamente con el Dios cristiano, de aquí al cielo y sería justamente este espacio el que se pensaría como no social, sin cultura en el sentido de sin normas de convivencia humana.

En este sentido la tensión entre pampa/ch'uru referida a lo salvaje o desorden que provoca la anomía o lo no cultivado por las normas y las reglas en la vida humana versus el orden de lo social, está presente en las clasificaciones y estas determinan su confección y su uso; pero aún resta por profundizar significados tanto en la confección de las propias fajas como en las otras piezas. Sin duda, la persistencia de las formas y de los diseños conducen a una estructura con escasas posibilidades de transformaciones. $\mathrm{La}$ boca o lak'a, las figuras que la acompañan llamadas linku y las k'isa (los colores que transitan del claro al oscuro y viceversa como conjuntos necesarios) que encierran un centro, son regulares. A pesar de los cambios, propios del entorno de las tejedoras, estos segmentos continúan presentes en la manufactura 
de las fajas, materializando relaciones que aún debemos investigar.

Agradecimientos: Este artículo forma parte del proyecto de Investigación Mayor en Ciencia y Tecnología de la Universidad de Tarapacá $\mathrm{N}^{\circ} 3712-14$. Se agradece el apoyo del Convenio de Desempeño Universidad de Tarapacá y Ministerio de Educación Pública. Agradecemos la contribución de los evaluadores del artículo.

\section{Referencias Citadas}

Arnold, D. 2007. Ensayos de los orígenes del textil. En Hilos Sueltos. Los Andes desde lo Textil, editado por D. Arnold, J.D. Yapita y E. Espejo, pp. 49-84. Plural e ILCA, La Paz.

Bertonio, L. 1984 [1612]. Vocabulario de la Lengua Aymara. Ediciones Ceres, Cochabamba.

Cereceda, V. 2010 [1977]. Semiología de los tejidos andinos. Las talegas de Isluga. Chungara Revista de Antropología Chilena 42:181-190.

Choque, P.G. 2002. Breve Diccionario: Aymara-Castellano, Castellano-Aymara: Aru pirwa. Ediciones Instituto de Estudios Andinos Isluga, Universidad Arturo Prat.

Fischer, E. 2011. Los tejidos andinos, indicadores de cambio: apuntes sobre su rol y significados en una comunidad rural. Chungara Revista de Antropología Chilena 43:267-282.

Franquemont, E.M., Ch. Franquemont y B.J. Isbel 1992. Awaq ñawin: El ojo del tejedor. La práctica de la cultura en el tejido. Revista Andina 10:47-80.
Gavilán, V. y L. Ulloa 1992. Proposiciones metodológicas para el estudio de los textiles andinos. Revista Andina 19:107-134.

Gisbert, T., S. Arce y M. Cajías 1987. Arte Textil y Mundo Andino. Gisbert y CIA S.A., La Paz.

Medvinski, D. 1977. Las Fajas de Isluga. Tesis de Licenciatura en Diseño, Escuela de Diseño, Universidad Católica de Chile, Santiago.

Silverman, G. 1988. Significado simbólico de las franjas multicolores tejidas en los wayakos de los Q'ero. Boletín de Lima 57:37-44.

Silverman, G.P. 1994. La metáfora del cuerpo humano. Anthropologica 63:65-85.

Torrico, C. 1989. Living weavings: the simbolism the Bolivian Herders's Sacks. Manuscrito en posesión de las autoras.

Zorn, E. 1987. Un análisis de los tejidos en los atados rituales de los pastores. Revista Andina 5:489-522.

\section{Notas}

1 El urdido se realiza llevando el hilo de estaca a estaca en forma de diagonal de ida y vuelta, formando un "ocho" al cruzarse los hilos. A esta vuelta entera de estaca a estaca formando un cruce, las tejedoras le llaman chinu. Puesto que la cuenta se hace en pares si hablamos de un chinu es igual a dos cruces, lo que equivale a cuatro cabos de urdimbre. Si estos cruces o chinu contienen cuatro, cinco o más cabos de diferentes colores, sigue configurándose como un chinu.
2 Comunicación personal (2013) Teresa Cañipa, profesional del Museo San Miguel de Azapa, Universidad de Tarapacá, Arica.

3 Se trata de una versión actualizada de Semiologie des Tissus andins: les talegas d' Isluga, publicada en 1978 por Annales E.S.C. vol. 33 (56):1017-1095.

4 Las mujeres de Cariquima tienden a nombrarlas como cuchu. 